\title{
Molecular Dynamics of a Smectic Liquid Crystalline Side-Chain Copolymer by Dielectric Relaxation Spectroscopy
}

\author{
Kazuo Araki \\ Department of Chemistry, Faculty of Science, Science University of Tokyo, \\ Kagurazaka, Shinjuku-ku, Tokyo 162, Japan
}

(Received July 6, 1989)

\begin{abstract}
KEY WORDS Liquid Crystal / Director Order Parameter / AC Electric Field /
\end{abstract}

Liquid crystalline side-chain polymers, as well-known, show promise as electro active or magnetro active materials for optical information storage and processing. A number of studies of these liquid crystalline polymers have been published. ${ }^{1-4}$ The motion of the dipole group in mesogenic side-chain may be observed and interesting results are obtained by dielectric relaxation spectra. ${ }^{5-15}$ These dielectric studies of side-chain polymers examined films which were aligned macroscopically using a combination of electrical and thermal treatment. Materials very similar to those in this paper have been reported to show piezoelectricity. ${ }^{16}$ In this paper we report dielectric behavior for this liquid crystalline side-chain copolymer.

The Smectic liquid crystalline side-chain copolymer used for our investigation was supplied from Kurary Co., Ltd., and has the following structure:

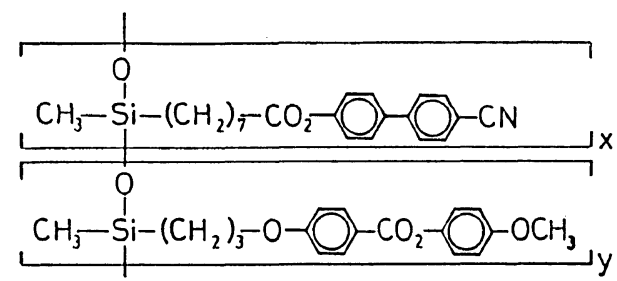

where $x: y=1: 3 ; x+y=40$. The glass transition, $T_{\mathrm{g}}$ and clearing temperature are 16 and $117^{\circ} \mathrm{C}$, respectively. The dielectric measurements were made in the range 25 to $10^{5} \mathrm{~Hz}$ using a GenRad 1689 Precision RLD Digibridge. The dielectric loss data are presented here as $G / \omega=\varepsilon^{\prime \prime} C_{a}$ where $G$ is the equivalent parallel conductance of sample, $\omega=2 \pi f / \mathrm{Hz}, \varepsilon^{\prime \prime}$ is the dielectric loss factor and $C_{a}$ is the interelectrode capacitance. The sample for dielectric measurement were prepared by cooling to room temperature at a rate of approximately $0.03 \mathrm{~K} \mathrm{~min}^{-1}$ from the isotropic phase. The details of the dielectric measurement are reported in a previous paper. ${ }^{9}$

Figures $1 \mathrm{a}$ and $1 \mathrm{~b}$ show the dielectric loss data at different temperatures above the glass transition temperature, $T_{\mathrm{g}}$. The dielectric loss peaks observed in Figure 1a are very broad from 28 to $42^{\circ} \mathrm{C}$ above $T_{\mathrm{g}}$ in the frequency range of about $100 \mathrm{~Hz}$ to $100 \mathrm{kHz}$, while those in Figure $1 \mathrm{~b}$ are fairly sharp curves in the temperature 52 to $92^{\circ} \mathrm{C}$. The half height width for the material is about 1.7 units of $\log _{10}$. This loss peak does not exhibit high frequency shoulder in the curve such as aligned smectic liquid crystalline polymer. ${ }^{10}$ Consequently, in this temperature this sample shows the typical overlapping $\alpha$ - and $\delta$-relaxation process, where $\alpha$-and $\delta$-relaxation processes reflect the motion of longitudinal or transverse component dipole moment of mesogenic head group. These 


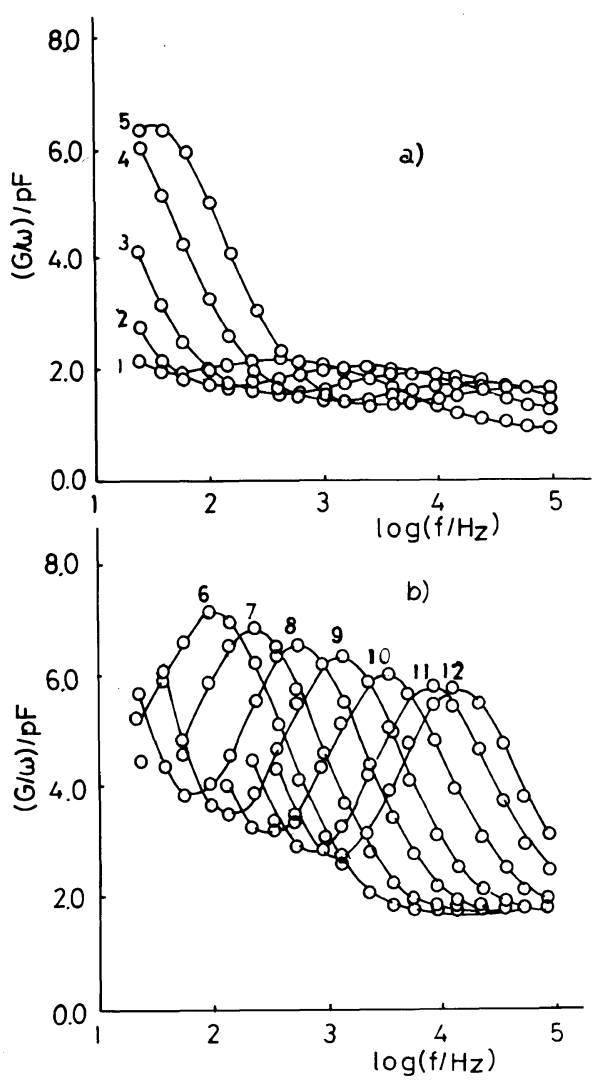

Figure 1. (a): Frequency dependence of the dielectric loss factor, $G / \omega$ in the temperature range 29.6 to $46.0^{\circ} \mathrm{C}$. Curves $1-5$ correspond to $29.6,34.1,37.8,42.1$, and $46.0^{\circ} \mathrm{C}$. (b): Temperature range 53.7 to $92.5^{\circ} \mathrm{C}$. Curves $6-12$ correspond to $53.7,57.7,65.5,71.5,79.4,89.0$, and $92.5^{\circ} \mathrm{C}$.

results are similar to those for the smectic siloxane copolymer. ${ }^{14}$ The dielectric loss curves in Figure $1 \mathrm{~b}$ are in the case of heating process from 52 to $92^{\circ} \mathrm{C}$. As shown in Figure 1b, the magnitude of dielectric loss factor decreases with temperature increase. However, they increase by cooling from 92 to $52^{\circ} \mathrm{C}$.

The Arrhenius plots for two temperature ranges are shown in Figure 2. The apparent activation energies for $\alpha$ - and $\delta$-processes were $198.0 \mathrm{~kJ} \mathrm{~mol}^{-1}$ and $121.6 \mathrm{~kJ} \mathrm{~mol}^{-1}$, respectively. Generally, a value of about $200 \mathrm{~kJ}$ $\mathrm{mol}^{-1}$ is indicative of an $\alpha$-relaxation process $^{17}$ which is commonly seen for this relaxation in amorphous glass-forming materials

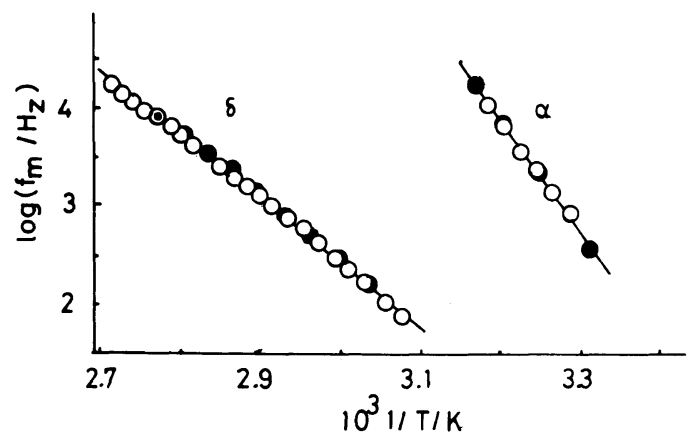

Figure 2. Arrhenius plots for the dielectric relaxation frequencies of $\alpha$ - and $\delta$-process. (O), heating; (O), cooling.

and is associated with micro-brownian motion of the dipole groups along the chain. However, the mechanism for this relaxation in the liquid crystalline polymer should differ from that in amorphous polymeric and nonpolymeric glass forming materials. The dipole reorientation must be constrained by the liquid crystal potential of the amorphous phase. A value of about $120 \mathrm{~kJ} \mathrm{~mol}^{-1}$ for $\delta$ process, which is $\varepsilon_{\|}$-relaxation by rotation of the mesogenic group around the backbone chain, is reasonable. As shown in figures, the values of maximum loss factor and the apparent activation energies for cooling process were quite the same as those of heating process.

In Figure 3, we show representative loss data obtained for the partially aligned samples with $100 \mathrm{~V} \mathrm{rms} / 1 \mathrm{kHz}$, partially aligned with $300 \mathrm{~V}$ $\mathrm{rms} / 10 \mathrm{kHz}$ and unaligned. The samples were prepared by cooling from isotropic phase to room temperature at a rate of approximately $0.03 \mathrm{~K} \mathrm{~min}^{-1}$ in the presence of a.c. electric field, and then heating. In this figure an isosbestic point is clearly seen at 3.6 units of $\log _{10}$ (frequency) as detailed in previous paper. ${ }^{13}$ It is possible to evaluate the director order parameter, $S_{\mathrm{d}}$ of two samples shown in Figure 3. In a homeotropically aligned mesophase the director and aligning electric field direction are parallel, i.e., $n / / E$, and the director order parameter $S_{\mathrm{d}}$ is unity. For a 


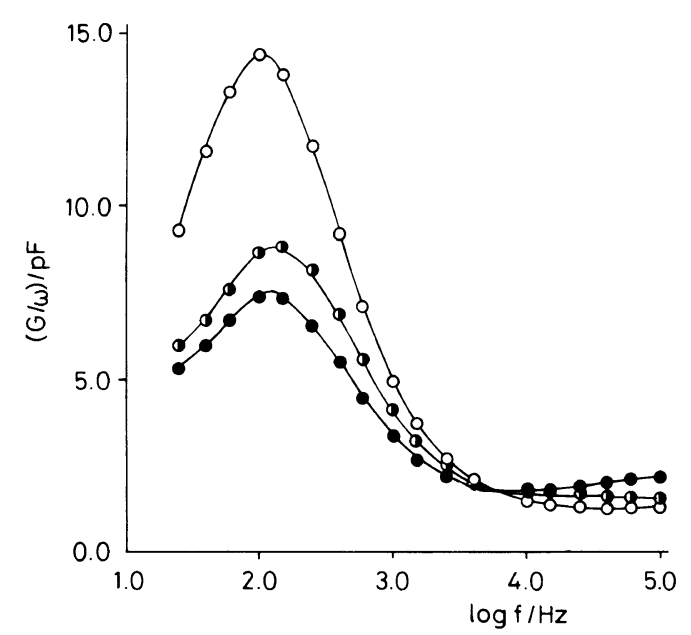

Figure 3. The dielectric loss factor, $G / \omega$ for partially aligned samples with $100 \mathrm{~V} \mathrm{rms} / 1 \mathrm{kHz}(O)$, partially aligned with $300 \mathrm{~V} \mathrm{rms} / 1 \mathrm{kHz}(\mathbf{O})$ and unaligned (O). The sample temperature was $52.3^{\circ} \mathrm{C}$.

planarly aligned mesophase, the director and the aligning electric field direction are perpendicular, i.e., $n \perp E$ and the director order parameter is -0.5 . For unaligned materials, the director order parameter is zero. The director order parameter $S_{\mathrm{d}}$ for a partially aligned sample is expressed using loss factor, $\varepsilon_{\mathrm{PA}}^{\prime \prime}$ as following

$$
\varepsilon_{\mathrm{PA}}^{\prime \prime}=(1 / 3) \varepsilon_{\|}^{\prime \prime}\left(1+2 S_{\mathrm{d}}\right)+(2 / 3) \varepsilon_{\perp}^{\prime \prime}\left(1-S_{\mathrm{d}}\right)
$$

where the $S_{\mathrm{d}}$ is related to $\left\langle\cos ^{2} \theta\right\rangle$ by the relation $\left(3\left\langle\cos ^{2} \theta\right\rangle-1\right) / 2$. For an unaligned sample, $S_{\mathrm{d}}$ is zero, so that, eq 1 becomes deduced expression.

$$
\varepsilon_{\mathrm{U}}^{\prime \prime}=(1 / 3) \varepsilon_{\|}^{\prime \prime}+(2 / 3) \varepsilon_{\perp}^{\prime \prime}
$$

Since eq 1 and 2 are valid at any frequency, we may compare loss values at any frequency to work out the $S_{\mathrm{d}}$. In this study we extracted the process in a sample with a different degree of alignment (see in Figure 3). Consequently, $\varepsilon_{\perp}^{\prime \prime}$ are absent in $\varepsilon_{\delta}$ the $\delta$-processes for partially aligned and unaligned samples, i.e., $\varepsilon_{\perp}^{\prime \prime}=0$. Therefore, $\varepsilon_{\mathrm{PA}, \delta}^{\prime \prime}$ may be written from eq 1 and 2 are follows,

$$
\varepsilon_{\mathrm{PA}, \delta}^{\prime \prime}=(1 / 3)\left(1+2 S_{\mathrm{d}}\right) \varepsilon_{\|, \delta}^{\prime \prime}=\left(1+2 S_{\mathrm{d}}\right) \varepsilon_{\mathrm{U}, \delta}^{\prime \prime}
$$

where $\varepsilon_{U, \delta}^{\prime \prime}$ is $\varepsilon_{\|, \delta}^{\prime \prime} / 3$. Consequently, for a full aligned sample loss factor $\varepsilon_{\delta}^{\prime \prime}$ is three times that of the unaligned sample. The ratio of strengths for the process in partially aligned and unaligned samples using the Fuoss-Kirkwood's parameter, ${ }^{17} \beta$ leads to eq 4 .

$$
\Delta \varepsilon_{\mathrm{PA}, \delta}^{\prime \prime} / \Delta \varepsilon_{\mathrm{U}, \delta}^{\prime \prime}=\left(\varepsilon_{\mathrm{m}}^{\prime \prime} / \beta\right)_{\mathrm{PA}, \delta} /\left(\varepsilon_{\mathrm{m}}^{\prime \prime} / \beta\right)_{\mathrm{U}} .
$$

In this study $\delta=$ processes in partially aligned and unaligned samples had the same frequency of maximum loss factor, $f_{\mathrm{m}}$ and the same value of the Fuoss-Kirkwood's parameter, $\beta$, i.e. $\beta_{\mathrm{PA}, \delta}=\beta_{\mathrm{U}, \delta}$. Thereofre eq 4 is given by

$$
\Delta \varepsilon_{\mathrm{PA}, \delta}^{\prime \prime} / \Delta \varepsilon_{\mathrm{U}, \delta}^{\prime \prime}=\left(\varepsilon_{\mathrm{m}}^{\prime \prime}\right)_{\mathrm{PA}, \delta} /\left(\varepsilon_{\mathrm{m}}^{\prime \prime}\right)_{\mathrm{U}, \delta}=1+2 S_{\mathrm{d}}
$$

Therefore, from eq 3-5, the ratio of maximum loss factor for partially aligned and unaligned sample leads to

$$
S_{d}=(1 / 2)\left[\left(\varepsilon_{\mathrm{m}}^{\prime \prime}\right)_{\mathrm{PA}, \delta} /\left(\varepsilon_{\mathrm{m}}^{\prime \prime}\right)_{\mathrm{U}, \delta}-1\right]
$$

This equation is the same relation to that obtained by Attard and Williams ${ }^{10}$ (see in eq 16 in ref 10 ). In our case, $\varepsilon^{\prime \prime}$ is related to conductance $G$, i.e., $G / \omega=\varepsilon^{\prime \prime} C_{a}$, where $C_{a}$ is the geometric capacitance of the space occupied by the sample and $\omega$ is the angular frequency $2 \pi f$. So we evaluated the director order parameter $S_{\mathrm{d}}$ from the value of $G / \omega$ in stead of $\varepsilon^{\prime \prime}$. The values of the maximum loss factors, $(G / \omega)_{\max }$ for the partially aligned sample with electric field $100 \mathrm{~V} \mathrm{rms} / 1 \mathrm{kHz}$ and $300 \mathrm{~V} \mathrm{rms} / 1 \mathrm{kHz}$ are 13.54 and 7.92, respectively, and then that of unaligned sample is 6.17. Therefore, the director order parameter $S_{\mathrm{d}}$ for the first and second sample from eq 6 were 0.60 and 0.11 , respectively. It was mentioned above that eq 6 should be equal to the relation obtained from Attard and Williams. ${ }^{10}$ So we examined the justification of eq 6 . If the materials used to study are incompletely alignment, some different relaxation processes should be observed in each main orientation. In this measurement range, only two relaxation regimes, $\alpha$ - and $\delta$-, have hertherto been found for the liquid 

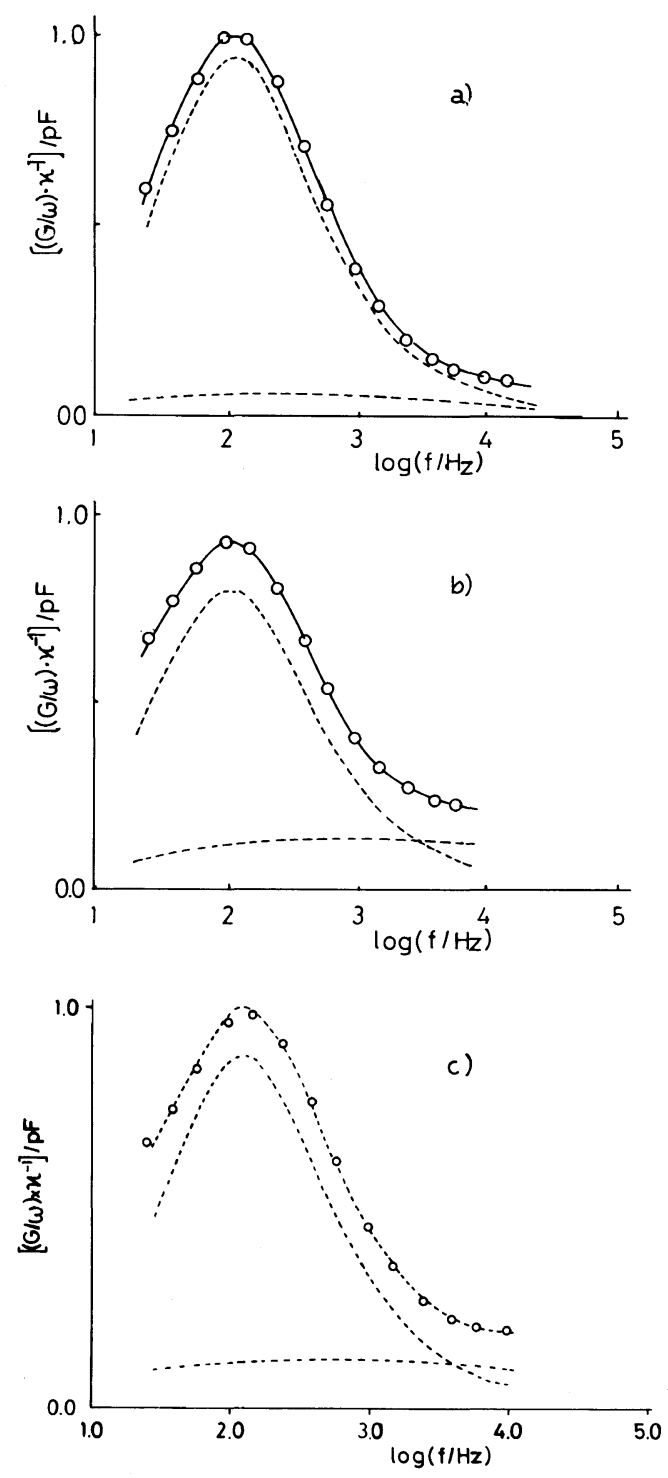

Figure 4. Representative sample of fits to the experimental loss data at $52.3^{\circ} \mathrm{C}$ in the smectic range for degrees of alignment. $\kappa$ is a scaling factor. (a) aligned with $100 \mathrm{~V}$ $\mathrm{rms} / 1 \mathrm{kHz}(\mathrm{b})$ aligned with $300 \mathrm{~V} \mathrm{rms} / 1 \mathrm{kHz}$ (c) unaligned sample. Solid and dotted lines are fitted curves according to eq 7 .

crystalline polymer. The dynamic process is expressed by the Fuoss-Kirkwood empirical relaxation function so that this relation is written as,
Table I. Fit parameters for loss curves shown in Figure 4

\begin{tabular}{lrrr}
\hline Samples & \multicolumn{1}{c}{$a_{\delta}$} & $\beta_{\delta}$ & \multicolumn{1}{c}{$A_{\delta}$} \\
\hline Unaligned & 6.17 & 0.76 & 8.12 \\
Partial aligned, 1 & 13.54 & 0.78 & 17.36 \\
$\quad(100 \mathrm{~V} \mathrm{rms} / 1 \mathrm{kHz})$ & & & \\
$\begin{array}{c}\text { Partial aligned, } 2 \\
(300 \mathrm{~V} \mathrm{rms} / 1 \mathrm{kHz})\end{array}$ & 7.92 & 0.80 & 9.90 \\
\hline
\end{tabular}

$$
\begin{aligned}
G / \omega= & a_{\delta} \operatorname{sech}\left[\beta_{\delta} \ln \left(f / f_{\mathrm{m}, \delta}\right)\right] \\
& +a_{\alpha} \operatorname{sech}\left[\beta_{\alpha} \ln \left(f / f_{\mathrm{m}, \alpha}\right)\right]
\end{aligned}
$$

where $a_{i}$ is $(G / \omega)_{\max }$ for process $i$, which $i=\alpha$ and $\delta, \beta$ is the distribution parameter and $f_{\mathrm{m}, i}$ is the relaxation frequency. It is possible to estimate the dielectric increment $\Delta \varepsilon^{\prime \prime}$ for the entire relaxation using the Fuoss-Kirkwood's parameters $\beta_{i}$ and $\alpha_{i}$. Therefore, the dielectric increment $\Delta \varepsilon^{\prime \prime}$ is

$$
\left(\Delta \varepsilon^{\prime \prime}\right)_{i}=2\left(\varepsilon_{\mathrm{m}}^{\prime \prime} / \beta\right)_{i}=(2 / C a)\left(a_{i} / \beta_{i}\right)
$$

where $\left(\Delta \varepsilon^{\prime \prime}\right)_{i}$ for process $i$ is proportional to $A_{i}=a_{i} / \beta_{i}$, where $i$ is $\alpha$ or $\delta$. According to Attard and Williams, ${ }^{10}$ the relation between the director order parameter $S_{\mathrm{d}}$ and the ratio of $\left(A_{\mathrm{PA}, \delta}\right) /\left(A_{\mathrm{U}, \delta}\right)$ is given by

$$
\left(A_{\mathrm{PA}, \delta}\right) /\left(A_{\mathrm{U}, \delta}\right)=1+2 S_{\mathrm{d}}
$$

This relation is quite the same as eq 5 . Figures $4 a, b$, and c show fitted curves according to eq 8 for $100 \mathrm{~V} \mathrm{rms} / 1 \mathrm{kHz}, 300 \mathrm{~V} \mathrm{rms} / 1 \mathrm{kHz}$ and unaligned sample, respectively. The fitted parameters for three samples are summarized in Table I. The director order parameter $S_{\mathrm{d}}$ obtained from eq 9 are 0.57 and 0.11 for the partially aligned sample with $100 \mathrm{~V} \mathrm{rms} / 1 \mathrm{kHz}$ and $300 \mathrm{~V} \mathrm{rms} / 1 \mathrm{kHz}$, respectively. These values are in fair agreement with those obtained from eq 7. Thus, from this fact, the distribution parameter for this process is the same value, so that $S_{\mathrm{d}}$ can be determined directly from the ratio of the strength of the loss factor.

Acknowledgment. The author thanks Dr. M. Matsumoto at Central Research Labor- 
atories, Kuraray Co., Ltd., for providing the sample.

\section{REFERENCES}

1. Ch. K. Ober, L. I. Jin, and R. W. Lenz, Adv. Polym. Sci., 59, 103 (1984).

2. M. G. Dobb and J. E. Mcintire, Adv. Polym. Sci., 60/61, 000 (1984).

3. K. Iimura, N. Koide, and M. Takeda, in "Current Topics in Polymer Science,” Vol. 1, R. H. Ottenbrite, L. A. Utracki and S. Inoue, Ed., Hauser Publishers, München, 1987, p 278.

4. N. Koide, Mol. Cryst. Liq. Cryst., 139, 47 (1986).

5. R. Zental, G. R. Strobl, and H. Ringsdorf, Macromolecules, 189, 60 (1985).

6. H. Kresse and V. Talrose, Makromol. Chem. Rapid Commun., 2, 369 (1981).

7. H. Kresse, S. Kostromin, and V. P. Shivaev, Makromol. Chem. Rapid Commun., 3, 509 (1982).

8. W. Haase, H. Pronoto, and F. J. Bormuth, Ber. Burs.
Phys. Chem., 89, 1229 (1985).

9. G. S. Attard, G. Williams, G. W. Gray, D. Lacey, and P. A. Gemmel, Polymer, 27, 185 (1986).

10. G. S. Attard and G. Williams, Liquid Cryst., 1253 (1986).

11. K. Araki and G. S. Attard, Liquid Cryst., 1, 301 (1986); Mol. Cryst. Liq. Cryst., 141, 69 (1986).

12. G. S. Attard, J. J. Moura-Ramos, and G. Williams, J. Polym. Sci., Polym. Phys., 25, 1099 (1987).

13. G. S. Attard, K. Araki, and G. Williams, Br. Polym. J., 19, 119 (1987); J. Mol. El., 3, 1 (1987).

14. G. S. Attard, J. J. Moura-Ramos, G. Williams, G. Nestor, M. S. White, G. W. Gray, D. Lacy, and K. J. Toyne, Makromol. Chem., 188, 2769 (1987).

15. G. S. Attard, K. Araki, J. J. Moura-Ramos, and G. Williams, Liquid Cryst., 3, 861 (1988).

16. K. Sato, K. Otsuka, and M. Matsumoto, Makromol. Chem. Rapid Commun., 9, 631 (1988).

17. N. G. McCrum, B. E. Read, and G. Williams, "Anelastic and Dielectic Effects in Polymeric Solids," John Wiley, London and New York, 1967. 